\title{
Coordination of bowing and fingering in violin playing
}

\author{
Andreas P. Baader ${ }^{\mathrm{a}}$, Oleg Kazennikov ${ }^{\mathrm{b}}$, Mario Wiesendanger ${ }^{\mathrm{c}, \mathrm{d}, *}$ \\ ${ }^{a}$ Department of Physiology, University of Berne, Switzerland \\ ${ }^{\mathrm{b}}$ Institute of Problems of Information Transmission, Russian Acad. Sci., Russia \\ ${ }^{\mathrm{c}}$ Department of Neurology, University of Berne, Switzerland \\ d. Department of Physiology, Neurophysiology Section, University of Fribourg, Rue du Musée 5, CH-1700 Fribourg, Switzerland
}

\begin{abstract}
Playing string instruments implies motor skills including asymmetrical interlimb coordination. How special is musical skill as compared to other bimanually coordinated, non-musical skillful performances? We succeeded for the first time to measure quantitatively bimanual coordination in violinists playing repeatedly a simple tone sequence. A motion analysis system was used to record finger and bow trajectories for assessing the temporal structure of finger-press, finger-lift (left hand), and bow stroke reversals (right arm). The main results were: (1) fingering consisted of serial and parallel (anticipatory) mechanisms; (2) synchronization between finger and bow actions varied from - 12 ms to $60 \mathrm{~ms}$, but these 'errors' were not perceived. The results suggest that (1) bow-finger synchronization varied by about $50 \mathrm{~ms}$ from perfect simultaneity, but without impairing auditory perception; (2) the temporal structure depends on a number of combinatorial mechanisms of bowing and fingering. These basic mechanisms were observed in all players, including all amateurs. The successful biomechanical measures of fingering and bowing open a vast practical field of assessing motor skills. Thus, objective assessment of larger groups of string players with varying musical proficiency, or of professional string players developing movement disorders, may be helpful in music education.
\end{abstract}

Theme: Control of posture and movement

Topic: Cognition

Keywords: Music; Bimanual coordination; Human; Motor control; Finger movements

\section{Introduction}

In primates, including man, most ecologically relevant skills require spatially and temporally coordinated actions of both hands. Typically, the individual limbs are engaged together in an asymmetric action for goal achievement. Previously, we have studied bimanual coordination in subjects opening a drawer with the left hand and manipulating a small object in the drawer recess with the right hand. Each subject made a series of pull-and-pick movements. Such manipulations are performed in everyday life;

* Corresponding author. Department of Physiology, Neurophysiology Section, University of Fribourg, Rue du Musée 5, CH-1700 Fribourg, Switzerland. Fax: +41316323011.

E-mail address: neuro@freesurf.ch (M. Wiesendanger). accordingly, all subjects found this an easy task, and needed only a few trials to familiarize themselves $[19,27,36]$. Characteristically, the two hands were synchronized at the goal, with a small (50-100 ms) pull-hand advantage over the pick-hand. In these studies, we have learned that virtually each single movement was executed somewhat differently in the sequence whereas goal-reaching was relatively invariant. This has been found to be typical for ecological motor behavior, for example, Refs. $[1,21]$.

We now report on the bimanual musical skill of violinplayers with varying experience. Expert playing of music requires most intricate, fast finger and bow movements that are organized in a prescribed ordinal and temporal sequence. In contrast to everyday bimanual actions, the goal of music performance lies in its auditory perception, with all its ingredients of rhythm, syntax, and emotional feelings [12]. 
Motor control studies in music are rare, and in string instruments, the issue of bimanual synchronization has not been approached. Research in motor control of music execution is problematical because of the imposed technical constraints. In string players, a few reports on bowing (without fingering) already revealed some of the complexities in the unimanual task [32,35], for example, constant-velocity bow strokes [24], or the coordination of wrist, elbow, and shoulder for rectilinear bow strokes [8]. Winold et al. [38] made a bowing study in professional cellists while playing short fragments of Brahms and Schubert. They observed that elbow and wrist movements were more variable than bow strokes and that fast movements were less variable and more constrained. Accentuations, especially at the beginning of a down-stroke, consisted of prominent acceleration peaks, visualized in phase-plane plots.

The present study provides a first account on simultaneous recordings of finger actions and bowing movements in violinists who had to play a simple music sequence at 4 metronome-paced tempi. The objectives were: (1) to clarify how independently the left-hand fingers are set in motion for tone production; (2) to assess mechanisms of fingering that determine the timing of tone onset and duration; (3) to quantify synchronization between fingers and bow actions. The approach used in this study was to assess basic and elementary motor prerequisites for playing the violin. The study of progressive skill acquisition of violinists would clearly require a higher number of subjects, amateurs as well as professionals. Also, it would be desirable to gauge the results with estimates of practice hours per year. Nevertheless, preliminary observations have shown that musical pieces of variable difficulty from various composers (Kreutzer, Valentini, Corelli, Bach) can be adequately recorded with the same methodology [37].

Parts of the results were presented in a poster [3].

\section{Methods}

\subsection{Movement analysis}

\subsubsection{Subjects}

Six violinists of varying skills participated in the study: $1=\mathrm{NE}$ (professional player and leader of an ensemble of virtuose players), $2=\mathrm{RH}$ (amateur without practice for several years), $3=\mathrm{MV}$ (amateur player, over 50 years of continuous practice), $4=\mathrm{PW}$ (part-time student with 8 years of violin education and practice), $5=\mathrm{SC}$ (amateur, moderate practice during about 4 years), $6=\mathrm{RS}$ (amateur, regular 6 years practice).

\subsubsection{Recordings}

Movements of the left-hand fingers II-V and of the bow (right hand) were recorded by means of a video-based, infrared-sensitive movement analysis system (ELITE strobe
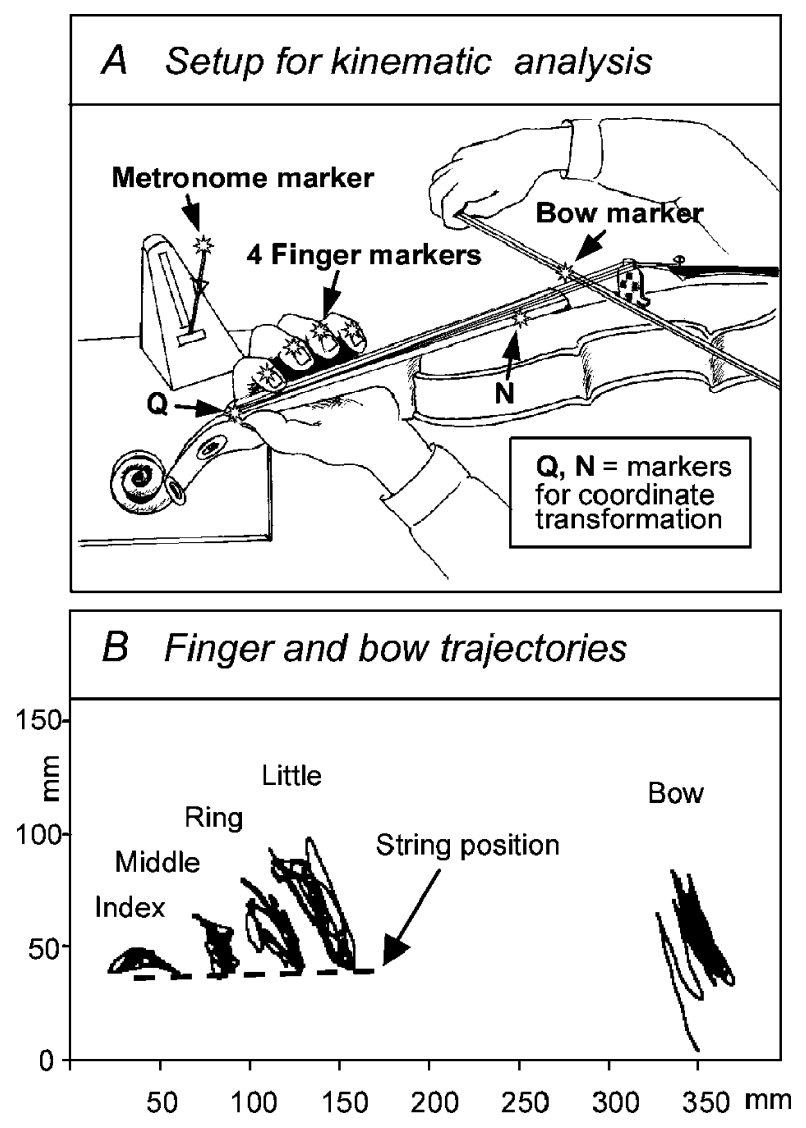

Fig. 1. (A) Positions of the 8 infrared reflecting markers (stars): one on each dorsal endphalanges of index, middle, ring and little fingers, the others on the wooden part of the bow, at the distal $(\mathrm{Q})$ and proximal $(\mathrm{N})$ parts of the fingerboard, and on the metronome pendulum. (B) Trajectories from the four fingers and the bow. The camera was set at an angle to reveal the largest finger excursions. The fingers point to the quasi-linear string position.

camera, 2D, $100 \mathrm{~Hz}$ ). Reflecting markers were fastened dorsally to the end-phalanx of the fingers and to the midportion of the bow (Fig. 1A). Additional markers were attached to the metronome pendulum (reference time signal) and to the proximal and distal ends of the fingerboard. Distance and position of the camera were chosen to obtain the largest finger excursions (Fig. 1B). The performance, together with the sound track, was also recorded with a conventional video system.

\subsubsection{Task}

Subjects played a sequence of 21 tones at 4 metronome-paced speeds $(110,140,160,180$ beats $/ \mathrm{min})$. The chosen tone sequence with the involved finger actions (Fig. 2, bottom panel) was deliberately simple and excluded string and positional changes (translations of the hand along the fingerboard). It covered 4 intervals from lower to higher pitches and vice versa. The sequence involved all 4 fingers and was repeated 10 times for all tempi. Each note was played without vibrato and with a separate (détaché) bow stroke in the first position on the D-string. 


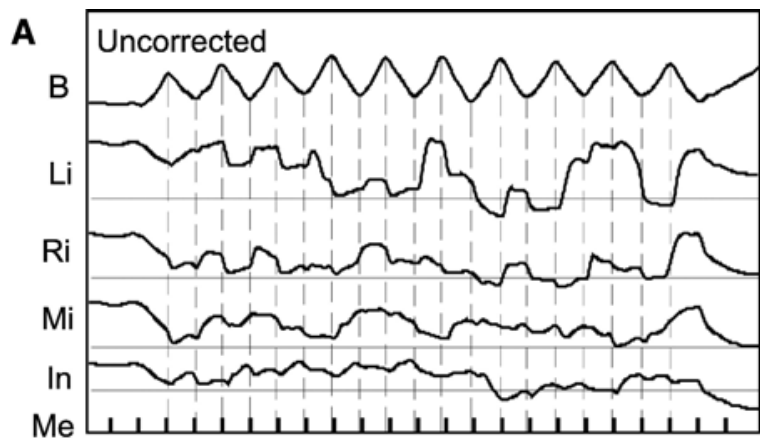

\section{B}
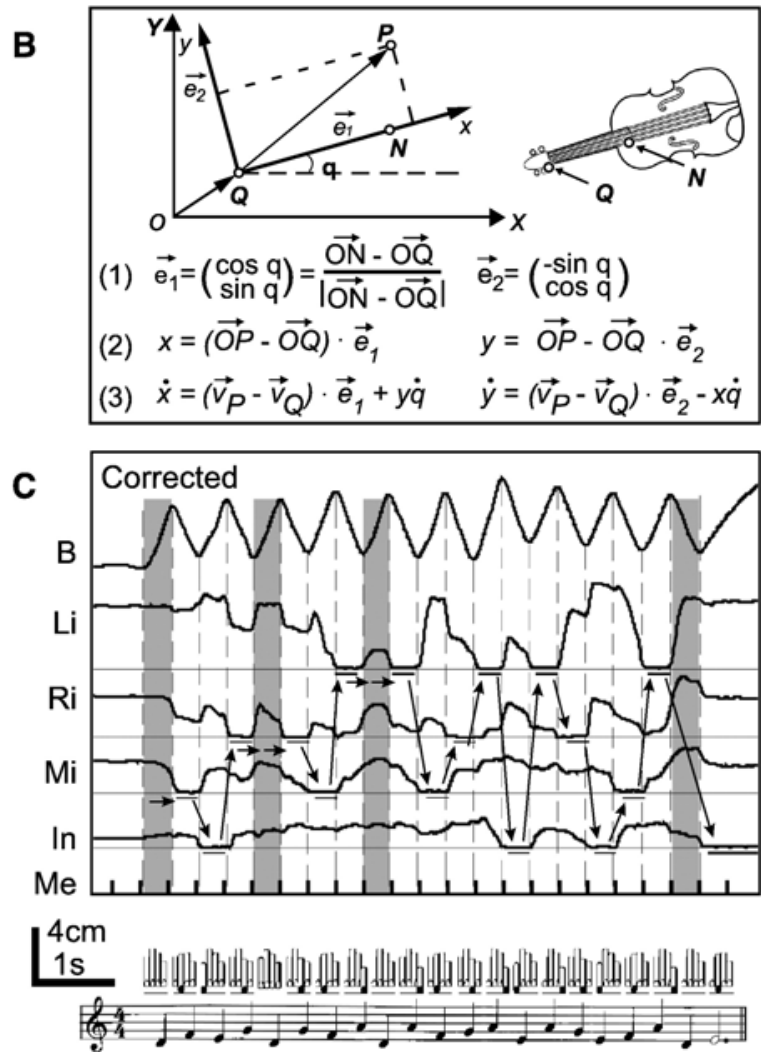

Fig. 2. (A) Original displacement traces of the bow movements (B) and the individual finger movements, containing additional components of instrument and body movements. $\mathrm{Me}=$ metronome; vertical dashed lines = instance of bow reversal. (B) Transformation of positional data from laboratory $(\mathrm{X} / \mathrm{Y})$ into violin-centered $(\mathrm{x} / \mathrm{y})$ coordinates. $\mathrm{O}$, Q: origins of the 2 coordinate systems with rotation in relation to lab space and their transformation through unit vectors (1) into position (2) and velocity (3). $\overrightarrow{\mathrm{OQ}}$ is the origin of the violin's coordinates, and $\theta(t)$ is the instantaneous angle between the violin $x$ axis and the laboratory system $x$ axis. (C) Same displacement data as in A after transformation into violin-centered coordinates. Tones are produced when the fingers press the string down to the finger board (see flat horizontal segments coinciding with the line which marks the fingerboard/string position for each individual finger). Shaded vertical columns indicate tone D produced by the bow stroke with an empty string (i.e., all fingers lifted from the string). The sequence of arrows corresponds to the musical notation shown on the bottom. The action-fingers are marked by a black dot above each note.

\subsubsection{Data analysis}

During recordings, the movements of left-hand fingering and right- hand bowing were unavoidably superimposed on the trunk movements of the playing subjects
(Fig. 2A). To extract only the finger and bow movements, we transformed the captured room-centered positional data into instrument-centered coordinates (see legend of Fig. 2B). After transformation (Fig. 2C), tone producing finger actions (here termed action-finger) and tone duration could be identified easily by the short horizontal intervals that coincided with the dotted line indicating the string/ finger board position, in musical language called fingerstop. However, not all flat intervals coincided with this position; a finger could also show a short motionless period without string contact, as shown for example for the little finger in the initial part of Fig. 2C. Obviously fingers, particularly those next to the finger depressing the string, tend to move and pause together. This enslaving of fingers is characteristic for manipulatory actions [41]. It was only after coordinate transformation that one could unambiguously distinguish fingerstops from the flat segments not coinciding with the fingerboard. Following the sequence of fingerstops, the musical episode could thus be reconstructed (arrows in Fig. 2C). When the empty Dstring was played (i.e., without fingerstop), all fingers had to be lifted from the string, as shown for the four shaded segments.

Peak acceleration or the abrupt flattening in the finger displacement are possible time references for marking finger-string depression. However, it turned out that for automatic computer evaluation, the most reliable reference point was finger peak-velocity $t$ (finger $-v_{\max }$ ) that clearly marked the impact of the fingerstop at stringfingerboard contact (Fig. 3). Bow reversal $t\left(\right.$ bow $\left._{\text {rev }}\right)$ was taken as time marker for bowing. The synchronization

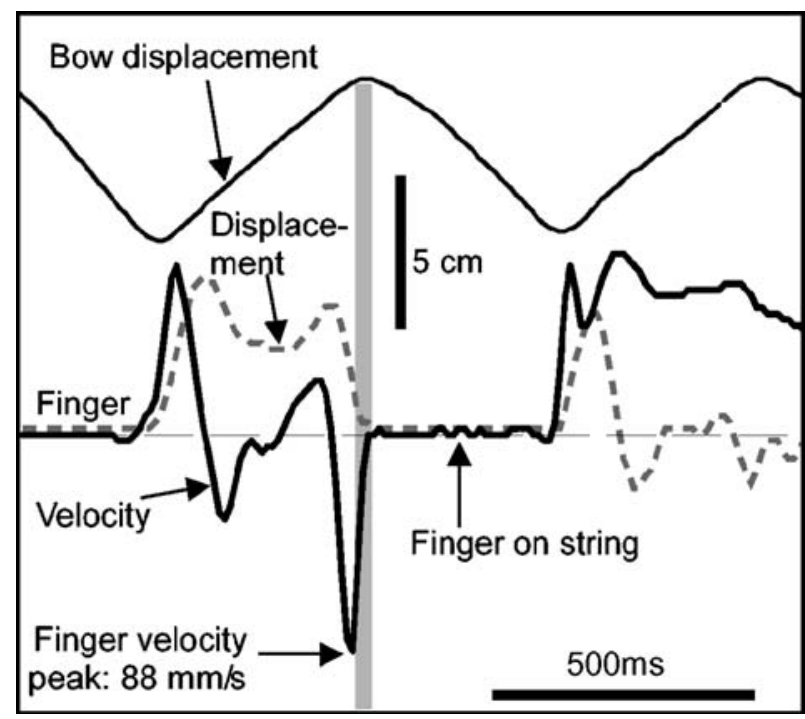

Fig. 3. Detailed view of bow, action-finger displacement, and action-finger velocity profiles. Note the abrupt decay of the velocity peak when the finger hits the string/fingerboard. This peak occurred at finger impact (left hand) and served as reference point of bow reversal (right hand). Both events were automatically detected, and the interval was taken to assess bimanual synchronization (gray shaded). Note that, in the displacement profile, the event of finger-string contact is less clear. 
interval $I_{\text {sync }}$ was thus calculated as difference of the two marker points

$I_{\text {sync }}=t\left(\right.$ finger $\left.-v_{\max }\right)-t\left(\right.$ bow $\left._{\text {rev }}\right)$

The bow movement in Fig. 3 was performed at slow pace and consisted of quasi-rectilinear (i.e., constant velocity) down- and upstrokes.

\section{Results}

\subsection{Consistency and the enslaving of fingering}

Subjects learned the simple sequence quickly and had not to read the score (notation). Within a subject, finger displacement profiles were consistent. This was representative for all subjects suggesting that a strong feedforward control determines the fingering and bowing sequence. Although the final phase of the finger towards the fingerboard was typically fast (see also Fig. 3 above) for depressing the string (in music called fingerstops), there were also consistent instances when finger movements toward the string were more gradual and slower. It occurred that the fast and the slow patterns were consistently related to certain tone sequences, even among subjects. These distinguishable patterns suggest that the slower movements toward the string have a functional meaning, as explained in the next section (see below).

\subsection{Serial versus parallel (anticipatory) finger actions}

In Fig. 4, 10 repetitions by the same subject are shown as superimposed traces of three fingers and the bow, recorded

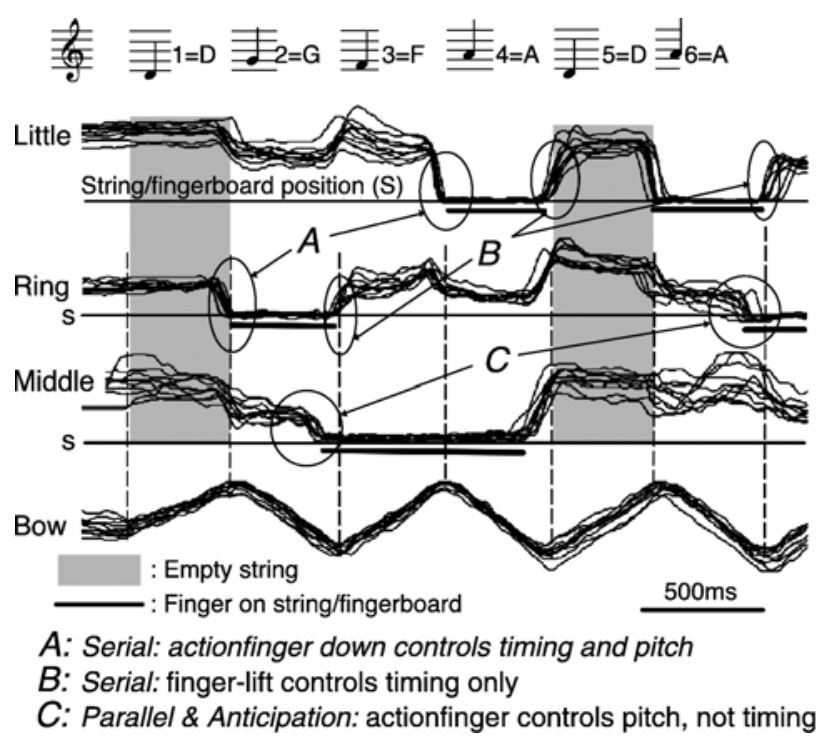

Fig. 4. One subject's repetitive playing of the basic melody is shown as displacement profiles with examples of serial and parallel-anticipatory mechanisms in fingering. Tone duration is indicated by vertical dashed lines, the tone sequence is shown at the top. The examples also demonstrate the combinatorial mechanisms of fingering and bowing. during a segment of 6 consecutive notes of the standard melody (D-G-F-A-D-A). It illustrates the main fingering mechanisms that contribute to the timing of the tone sequence. During the first interval, the bow stroke activated the 'empty' D-string, that is, all fingers were lifted from the string. During the next interval, the ring finger moved rapidly to the fingerboard playing the note $\mathrm{G}$. In this case, the actionfinger initiated the tone as determined by the impact of the action-finger on the string/fingerboard. The duration of the tone $\mathrm{G}$ was timed by the rapid lift of the action-finger. But, at the same instance, the lifting of the ring finger also initiated the next note, F, a lower note played on the same string. However, the new action-finger (middle finger) had reached the string before due time, that is, before the ongoing tone $G$ was finished. This is a typical, slower anticipatory motion pattern, performed by all subjects during a descending scale on the same string. The action of the middle finger determined the pitch of the tone F, but not its onset; the latter was triggered by the lifting of the ring finger above. In Fig. 4, pure serial events during the sequence (A, B), and anticipatory/parallel and combinatorial action in descending scales (C) are highlighted. Note that the anticipating action finger in $\mathrm{C}$ may remain on the string as long as another finger plays higher up on the string, preventing tone production below (see also 3rd interval F in Fig. 4). Both anticipatory/ parallel mechanisms and serial mechanisms can also be identified in Fig. 2C.

\subsection{Synchronization between the right bowing hand and left fingering hand}

In order to obtain many bimanual synchronization intervals, subjects had to play a new tone with each bow stroke. The timing of the fingering (finger impact on the string and finger lifts) was measured in relation to bow reversal. The interval-histograms are shown in Fig. 5, for finger-down actions and for two different speeds (144 beats/ min and 192 beats/min). In both histograms, the 4th subject from left (an amateur, but a good player) had unusually large synchronization intervals of 40 to $75 \mathrm{~ms}$, whereas the $3 \mathrm{rd}$ subject had values near zero. Synchronization intervals determined by finger lifts had mean values varying between 30 and $50 \mathrm{~ms}$.

The means were rather variable among subjects, possibly due to subtle variations in fingering strategies (e.g., different distances action fingers had to move for string contact). In contrast, SD values were more consistent among subjects and often did not vary with the means (see subject 4 with the largest mean but small SD values). Thus, the variability expressed by the SD appears to be a more valid measure of intermanual synchronization errors than the means. The small sample of subjects does not allow any conclusion about the relation of synchronization error and musical proficiency. An analysis of variance with repeated measures was performed for SD's, including the factor finger (the 4 involved fingers) and the factor speed of playing (metro- 

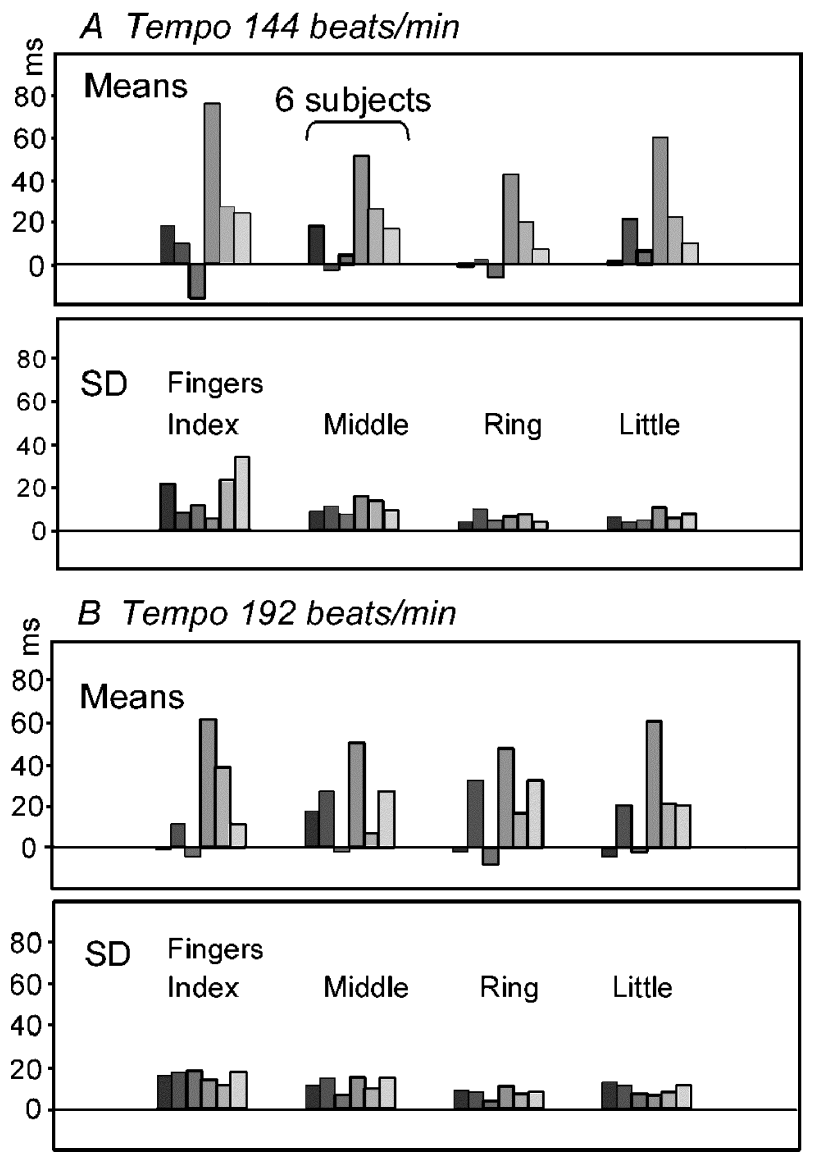

Fig. 5. Intervals between bowing and fingering at medium (A) and high (B) speed, plotted for the 4 fingers and for the 6 individual subjects. Mean positive values are the measure of finger-down actions relative to bow reversal, together with their standard deviations (SD). The tempo changes had no significant effects on synchronization. The SD were more uniform and did not change in parallel with the means.

nome $144 / \mathrm{min}$ and $192 / \mathrm{min})$. A significant main effect of the finger factor emerged $\left(F_{3,15}=10.4, P=0.00059\right)$, but not for the factor speed $\left(F_{1,5}=0.50908, P=0.5091\right)$. Interaction between fingers and speed was also not significant $\left(F_{3,15}=\right.$ $0.58176, P=0.63603)$.

\subsection{Anticipatory placements of action-fingers}

As mentioned before and illustrated in Fig. 4, anticipatory finger actions were characterized by their slow and irregular course toward the string. This occurred almost one tone in advance with the finger approaching slowly the fingerboard to make contact with the string. The results were long fingering-bowing intervals as shown in the histogram of Fig. 6. The anticipating finger determined only the pitch, not the timing of the tone and therefore could not be considered as a measure of synchronization. The histograms concern only three fingers since the little finger, being at the highest position on the string, could not be placed in anticipation below another finger (but note that playing over all strings would allow for similar anticipatory placing of an action-finger at bow-string changes).

\section{Discussion}

Our results provide the first simultaneously recorded biomechanical data about violin bowing and fingering. The goal was to understand the temporal coordination of bowing and fingering. In cognitive neuroscience, perception of music was emphasized in most previous studies $[17,26,40]$. Motor control studies were chiefly concerned with rhythmicity $[25,30]$ or pathological issues, like muscle cramps of professional musicians $[9,10,13]$. It was also reported that the brain of musicians acquires representations that differ from non-musicians. This can be taken as a model of longterm plasticity emerging from intensive musical practice from childhood onward over many years [23].

\subsection{Fingering mechanisms that determine the timing of a tone}

Humans move their fingers in relative isolation, termed finger individuation [18,31], ascribed to the emergence of monosynaptic cortico-motoneuronal connections [28]. However, a single muscle can be shared by two or more fingers that then become 'enslaved' [41]. Single finger selections during play also necessitate an inhibition of unwanted finger movements [4]. Our displacement profiles of left-hand fingers disclosed prominent and stereotypical coactivations of fingers, as seen also during typing [15] and piano playing [14]. However, only the tone-producing action-finger hits the string, whereas the 'unwanted' finger(s) stopped before string contact. This suggests that 'enslaving' of fingers is also under control of unknown mechanisms, for example, due to an inhibitory break, to an

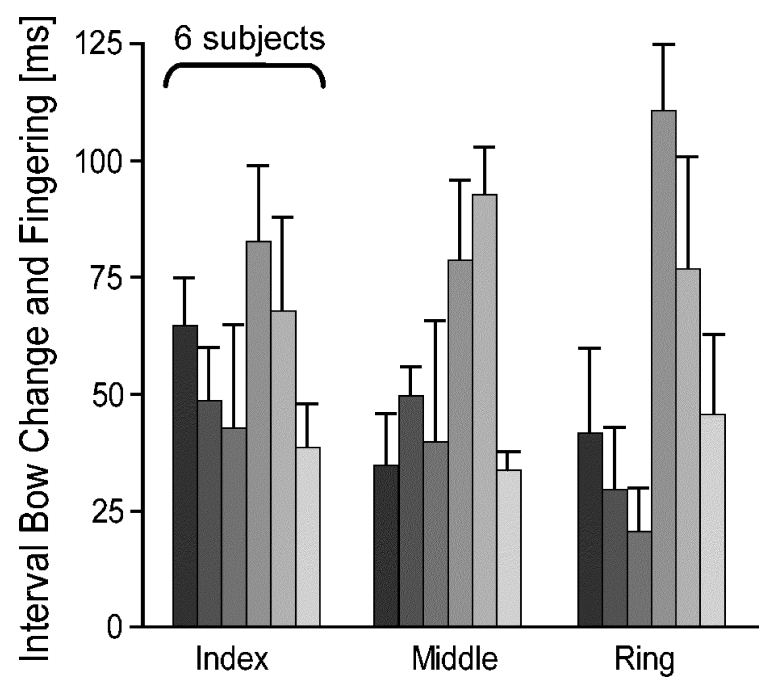

Fig. 6. Intervals between finger-string contact and bow reversal when the action finger moved slowly and in anticipation towards the fingerboard in a descending sequence. In this case, the action-finger determines the pitch only, but not tone onset and thus does not contribute to synchronization. This departure from a pure serial mechanism to an anticipatory finger placing was present in all subjects and invariably occurred in descending sequences. 
extension in the metacarpo-phalangeal joint, or to a smaller command signal.

Both, right-handed bowing and left-hand finger presses are a prerequisite for tone generation and contribute to tone initiation and termination (apart from other tone qualities, like loudness and timbre). During legato bow strokes, fingers, however, exclusively determine tone onset and duration. From the motor control point of view, playing a string instrument necessarily requires combinatorial actions of both limbs and also among the left-hand fingers. Finger taps and lifts, to and from the string, may initiate or terminate a tone. Or fingers can be placed more slowly on the string in anticipation. The start and the end of a tone may involve the action of two separate fingers. These mechanisms appear to be fully automatized since they were not consciously planned and were present in all subjects, suggesting that these automatisms develop quickly, also in amateurs.

\subsection{About the precision of synchronization between fingering and bowing}

In our paradigm, each tone of the sequence was played with a separate bow stroke (détaché bowing), and fingering was measured in relation to the instances of bow reversal. Trendelenburg, a physiologist who published a monograph on the practice of string instruments, clearly demonstrated that onset of a bow-movement preceded onset of stringvibration by about $40-50 \mathrm{~ms}$, depending on force and acceleration of bowing [34]. A similar imprecision is likely to occur also at bow reversal. Moreover, it was shown that the full frequency spectrum (defining the timbre) developed within some tenths of ms after bow reversal [11]. Ideally, the bow-specific timing should coincide with the finger actions. Given the above delay factors, it was not so surprising that, in the present experiments, we observed departures of about 30-60 ms and more from perfect synchronization. Lifting of a finger to initiate a tone tended to be slightly less synchronous with bow reversal. Probably, this is due to the fact that the lifting finger has to accelerate from zero, whereas the velocity peak occurred just at finger-string impact of the tapping (see Fig. 3). With respect to the mean intervals, we estimate that they are still in the range one would expect from the acoustic factors mentioned above. It appeared that the standard deviation (rather than the mean) better represents the temporal imprecisions in finger-bow coordination, particularly because the SD was not well associated with the mean (see subject 4 with highest mean). Our data revealed no correlation between the average departure from perfect synchronization and the proficiency of the players, notably with our best and professional player ranking in the middle of the group. In line with the present temporal imprecision, rhythmic hand clapping typically deviated by an unperceptible error of $30-50 \mathrm{~ms}$ from the metronome pacer [16]. In musical ensembles, the imprecisions among individual flute players were about $30 \mathrm{~ms}$, for brass players $39 \mathrm{~ms}$, and for string ensembles $51 \mathrm{~ms}$ [29].
The largest error in the string players was probably due to the bow actions [34]. Also, neural commands may be perfectly timed, but any movement, especially bowing, is fraught with inertial delays, which may not always be predicted precisely. The timing of bow reversal in professional cello players varied with a standard deviation of about $50 \mathrm{~ms}$ [38], which was about $2 \times$ higher than in the present study. Taken together, these various reports on tapping and music performance tend to be in line with our observation of some imprecision around $20 \mathrm{~ms}$ SD in bowfinger synchronization, not detectable by our ears and not disturbing auditory perception. Finally, it should be emphasized that the above temporal errors have to be distinguished from 'natural' and intentional fluctuations in tempo that are well-known ingredients of musical content and expression.

\subsection{Anticipation in fingering}

Whenever a finger was selected to depress the string in a descending sequence, the action finger was found to break away from the coactivation pattern, shifting to the individuation mode and resulting in a prolonged bow-finger synchronization (Fig. 6). This anticipatory move (occurring in parallel with the actual tone) was always associated with a rapid lifting of the finger above the string, thus controlling tone onset but not the pitch. The small number of subjects prevents an interpretation about the relation of anticipation with expertise, it rather suggests that it is a basic mechanism. In the present simple musical task, restricted on one string, anticipatory preparation occurred in descending tone sequences only. In less constrained conditions, similar proactive finger placements would also occur during string transitions or during scales that include whole-hand positional changes along the fingerboard. While anticipatory moves of the hand ('thumb under' phenomenon) were observed while playing ascending piano scales this was not the case when highly experienced typists performed one-handed sequences on the type-writer $[14,33]$. As noted by these authors, an anticipatory/parallel mechanism also occurs in language, termed 'co-articulation' [20], indicating that music and language share similar mechanisms. In summary, a tone sequence includes both, pure serial concatenation of finger movements and anticipatory/parallel features.

\subsection{Feedforward and feedback in motor control of music performance}

The present kinematic recordings revealed an impressive consistency in the smallest movement components on repetitive playing (Fig. 4). This may indicate that, at least for the very simple tone sequence, the control is limited to feedforward mechanisms. Lashley [22] argued that, in the rapid sequences of articulation, sensory control is unlikely to play a role; rapid sequences of course also occur in music. It is of interest, however, that single tactile afferents may encode delicate fingertip forces, as demonstrated by 
Birzniek et al. [5]. Furthermore, it was concluded that, during speech, "... sensorimotor mechanisms [are used] to update and adjust individual serial actions on a movementto-movement basis" [2]. A particularly difficult task in violin playing, requiring intensive practice, is to place the finger at the correct place on the smooth fingerboard for achieving the intended pitch (intonation). Although correct fingering may become gradually automatized during learning, one should think that finger sensibility would still be highly important in establishing and shaping central representations of touch and hearing. While auditory feedback obviously is of prime importance in playing music, there is little information about the role of tactile and proprioceptive cues. Objective documentations from musicians developing sensory disturbances are much desirable. We suggest that objective recordings of fingering movements will provide valuable insights into the pathophysiology of various disorders in string players, including tactile deficiencies.

Current theories about the role of feedback in the production of skilled movements go beyond the classical reflex-like corrections of motor output; they rather point to a decisive role in correcting central commands. In this view, the descending predictive signal (top-down) and the driving sensory feedback (bottom up) are used to adaptively adjust the sensorimotor representations or 'internal model', for example, $[6,7,39]$. This would refute the objection of Lashley [22] that fast serial actions, including music performance (and particularly the learning of a new piece of music) could notfor timing reasons - rely on somatosensory feedback.

In conclusion, we submit that the present novel investigation, although resting on a relatively small number of violin players, has potentially the power to investigate larger samples of musicians of varying proficiency in motor control, including the observed fingering and bowing mechanisms. But even studies based on more demanding musical excerpts than that used in this paper will not provide answers for the truly musical genius. The motor control approach deals with motor mechanisms and technical skills, that is, the prerequisites of musical proficiencies. However, it does not necessarily encompass true musicality.

\section{Acknowledgments}

We thank P. Milani, H.P. Marro and O. Amidzic for technical help in various periods of the work.

\section{References}

[1] J.H. Abbs, K.J. Cole, Neural mechanisms of motor equivalence and goal achievement, in: S.P. Wise (Ed.), Higher Brain Functions, Wiley, New York, 1987, pp. 15-43.

[2] J.H. Abbs, N.P. Connor, Motor coordination for functional human behaviors: perspectives from a speech motor data base, in: Perspectives on the Coordination of Movement, vol. 61, North Holland, Amsterdam, 1989, pp. 157-183.
[3] A.P. Baader, P. Milani, M. Wiesendanger, Bimanual coordination in violin players: synchronization and preparation of finger movements, in: Abstr. 28th Ann. Meet. Soc. Neurosci. Miami, vol. 25, 1999.

[4] K.M.B. Bennett, R.N. Lemon, Corticomotoneuronal contribution to the fractionation of muscle activity during precision grip in the monkey, J. Neurophysiol. 75 (1996) 1826-1842.

[5] I. Birznieks, P. Jenmalm, A.W. Goodwin, R.S. Johansson, Encoding of direction of fingertip forces by human tactile afferents, J. Neurosci. 21 (2001) 8222-8237.

[6] S.J. Blakemore, S.J. Goodbody, D.M. Wolpert, Predicting the consequences of our own actions: the role of sensorimotor context estimation, J. Neurosci. 15 (1998) 7511-7518.

[7] S.J. Blakemore, C.D. Frith, D.M. Wolpert, Spatio-temporal prediction modulates the perception of self-produced stimuli, J. Cogn. Neurosci. 11 (1999) 551-559.

[8] V. Braitenberg, The cerebellum and the physics of movement: some speculations, in: M. Glickstein, C. Yeo, J. Stein (Eds.), Cerebellum and Neuronal Plasticity, Plenum, New York, 1986, pp. 193-207.

[9] V. Candia, T. Elbert, E. Altenmüller, H. Rau, T. Schäfer, E. Taub, Constraint-induced movement therapy for focal hand dystonia in musicians, Lancet 353 (1999).

[10] V. Candia, T. Schaefer, T. Taub, H. Rau, E. Altenmüller, B. Rockstroh, T. Elbert, Sensory motor retuning: a behavioral treatment for focal hand dystonia of pianists and guitarists, Arch. Phys. Med. Rehabil. 83 (2002) $1342-1348$.

[11] W.A. Deutsch, Musik und computer, in: H. Bruhn, R. Oerter, H. Rösing (Eds.), Musikpsychologie. Ein Handbuch in Schlüsselbegriffen, Urban and Schwarzenberg, München, 1985, pp. 107-119.

[12] C. Drake, C. Palmer, Skill acquisition in music performance: relations between planning and temporal control, Cognition 74 (2000) 1-32.

[13] T. Elbert, V. Candia, E. Altenmüller, H. Rau, A. Sterr, B. Rockstroh, C. Pantev, E. Taub, Alteration of digital representations in somatosensory cortex in focal hand dystonia, NeuroReport 9 (1998) 3571-3575.

[14] K.C. Engel, M. Flanders, J.F. Soechting, Anticipatory and sequential motor control in piano playing, Exp. Brain Res. 113 (1997) 189-199.

[15] M. Flanders, J.F. Soechting, Kinematics of typing: parallel control of the two hands, J. Neurophysiol. 67 (1992) 1264-1274.

[16] P. Fraisse, Les synchronisations sensori-motrices aux rythmes, in: J. Requin (Ed.), Anticipation et Comportement, CNRS, Paris, 1980, pp. $233-257$.

[17] N. Griffith, P.M. Todd, Musical Networks-Parallel Distributed Perception and Performance, MIT Press, Cambridge, MA, 1999 (385 pp).

[18] C. Häger-Ross, M.H. Schieber, Quantifying the independence of human finger movements: comparisons of digits, hands, and movement frequencies, J. Neurosci. 15 (2000) 8542-8550.

[19] O. Kazennikov, S. Perrig, M. Wiesendanger, Kinematics of a coordinated goal-directed bimanual task, Behav. Brain Res. 134 (2002) 83-91.

[20] R.D. Kent, S.G. Adams, The concept and measurement of coordination in speech disorders, in: S.A. Wallace (Ed.), Perspectives on the Coordination of Movement, North Holland, New York, 1989, pp. $415-450$.

[21] K.S. Lashley, Basic neural mechanisms in behavior, Psych. Rev. 37 (1930) $1-24$

[22] K.S. Lashley, The problem of serial order in behavior, in: L. Jeffress (Ed.), Cerebral Mechanisms in Behavior, Interscience, New York, 1951, pp. $112-136$.

[23] T.F. Münte, E. Altenmüller, L. Jäncke, The musician's brain as a model of neuroplasticity, Nat. Rev., Neurosci. 3 (2002) 473-478.

[24] W.L. Nelson, Physical principles for economics of skilled movements, Biol. Cybern. 46 (1983) 135-147.

[25] C. Palmer, Music performance, Ann. Rev. Psychol. 48 (1997) $115-138$

[26] R. Parncutt, G.E. McPherson, The Science and Psychology of Music Performance-Creative Strategies for Teaching and Learning, Oxford Univ. Press, Oxford, 2002 (388 pp). 
[27] S. Perrig, O. Kazennikov, M. Wiesendanger, Time structure of a goaldirected bimanual skill and its dependence on task constraints, Behav. Brain Res. 103 (1999) 95-104.

[28] C.G. Phillips, R. Porter, Corticospinal neurones, their role in movement, in: Monogr. Physiol. Soc., vol. 34, Academic Press, London, 1977 (450 pp)

[29] R.A. Rasch, Timing and synchronization in ensemble performance, in: J.A. Sloboda (Ed.), Generative Processes in Music, Clarendon Press, Oxford, 1988, pp. 70-90.

[30] B.H. Repp, Control of expressive and metronomic timing in pianists, J. Mot. Behav. 31 (1999) 145-164.

[31] M.H. Schieber, Muscular production of individuated finger movements: the roles of extrinsic finger muscles, J. Neurosci. 15 (1995) 284-297.

[32] G. Shan, P. Visentin, A quantitative three-dimensional analysis of arm kinematics in violin performance, Med. Probl. Perform. Artists 18 (2003) 3-10.

[33] J.F. Soechting, A.M. Gordon, K.C. Engel, Sequential hand and finger movements: typing and piano playing, in: J.R. Bloedel, T.J. Ebner, S.P. Wise (Eds.), The Acquisition of Motor Behavior in Vertebrates, MIT Press, Cambridge, MA, 1996, pp. 343-360.
[34] W. Trendelenburg, Die natürlichen Grundlagen der Kunst des Streichinstrumentenspiels, Springer, Berlin, 1925 (300 pp).

[35] P. Visentin, G. Shan, The kinetic characteristics of the bow arm during violin performance: an examination of internal loads as a function of tempo, Med. Probl. Perform. Artists 18 (2003) 91-97.

[36] M. Wiesendanger, O. Kazennikov, S. Perrig, P. Kaluzny, Two handsone action, in: A.M. Wing, P. Haggard, R. Flanagan (Eds.), Hand and Brain, Oxford Univ. Press, Oxford, 1996, pp. 283-300.

[37] M. Wiesendanger, A.P. Baader, O. Kazennikov, A biomechanical approach to fingering and bowing in Violinists in: E. Altenmüller, J. Kesselring, M. Wiesendanger (Eds.), Music, Motor Control, and the Brain, Oxford Univ. Press, Oxford, in press.

[38] H. Winold, E. Thelen, D.B. Ulrich, Coordination and control in the bow arm movements of highly skilled cellists, Ecol. Psychol. 6(1994) 1-31.

[39] A.G. Witney, P. Vetter, D.M. Wolpert, The influence of previous experience on predictive motor control, NeuroReport 12 (2001) 649-653.

[40] R.J. Zatorre, I. Peretz, The Biological Foundations of Music, NY Acad. Sci., New York, 2001 (462 pp).

[41] V.M. Zatsiorsky, Z.M. Li, M.L. Latash, Enslaving effects in multifinger force production, Exp. Brain Res. 131 (2000) 187-195. 